Europhys. Lett., 76 (5), pp. 863-869 (2006)

DOI: $10.1209 / \mathrm{epl} / \mathrm{i} 2006-10348-5$

\title{
Phase coexistence in highly deuterated ferroelectric triglycine selenate: Landau description
}

\author{
F. J. Romero $\left(^{*}\right)$, M. C. Gallardo and J. Del Cerro \\ Departamento de Física de la Materia Condensada, Instituto Mixto de Ciencia \\ de Materiales CSIC-Universidad de Sevilla - Apartado 1065, 41080 Sevilla, Spain
}

received 5 June 2006; accepted in final form 3 October 2006

published online 27 October 2006

PACS. 65.40.-b - Thermal properties of crystalline solids.

PACS. 77.80.Bh - Phase transitions and Curie point.

PACS. 77.84.Fa - KDP- and TGS-type crystals.

\begin{abstract}
The phase transition in a 90\% deuterated sample of TGSe has been investigated by calorimetric and dielectric measurements. As a consequence of the presence of an internal crack in the sample, the transition takes place in two stages separated by an interval where the paraelectric and the ferroelectric phases coexist without latent heat contribution. From the latent heat, the specific heat excess and the Curie law for the dielectric constant, it is shown that the transition follows a 2-4-6 Landau potential, whose coefficients are determined. This potential describes completely all the data, including those corresponding to the coexistence interval.
\end{abstract}

Introduction. - Compositional changes affect very significantly most ferroelectric phase transitions and their effects are particularly important if the transition is close enough to a tricritical point, so that the first- or second-order nature could be modified. Triglycine selenate $\left[\left(\mathrm{NH}_{2} \mathrm{CH}_{2} \mathrm{COOH}\right)_{3} \mathrm{H}_{2} \mathrm{SeO}_{4}\right]$, here-after TGSe, is a well-known uniaxial ferroelectric material belonging to the triglycine sulphate (TGS) family. It undergoes a typical order-disorder phase transition at about $T \sim 295 \mathrm{~K}$, the space group of the lower-temperature phase being $\mathrm{P} 2_{1}$ and that of the higher-temperature phase $\mathrm{P} 2_{1} / \mathrm{m}$. [1,2]. Recently, we performed a calorimetric study on the phase transition showing that the specific heat of the ferroelectric phase follows a classical tricritical Landau potential whose coefficients have been determined [3].

The effect of deuteration on the dielectric properties of phase transition in TGSe has been previously investigated [4-6]. It has been found that the thermal hysteresis as determined from dielectric-constant measurements increases when the deuteration increases, which indicates that the transition becomes first order.

Concerning the thermal properties of partially deuterated TGSe, the specific heat and the latent heat of a $62 \%$ deuterated sample and under the effect of $\gamma$-irradiation have been measured $[7,8]$. The specific heat behaviour was found to follow the predictions of a 2-4-6 Landau potential and the latent heat was evaluated to be $168 \mathrm{~J} \mathrm{~mol}^{-1}$.

In a very recent paper [9], we reported a preliminary analysis of the specific heat and latent heat of a $90 \%$ deuterated sample of TGSe using the technique "Square Modulated Differential Thermal Analysis" (SMDTA) [10], which has been proved to be very useful in the investigation

$\left(^{*}\right)$ E-mail: fjromero@us.es 
of first-order phase transitions. The high sensitivity of the equipment and the very small temperature variation rate used to perform the measurements (lower than $0.1 \mathrm{~K} \mathrm{~h}^{-1}$ ) made it possible to observe in detail the kinetics of the transformation and to obtain information about growth or destruction of domains in ferroelastic and ferroelectric phase transitions $[11,12]$. In the case of the $90 \%$ deuterated sample of TGSe, an internal crack was found in the sample as a consequence of the increase in the fragility when the deuteration increases. Although this crack is not expected to affect bulk properties, it has a very important role in the evolution of the phase front and it was found that the transformation takes place in two steps separated by an intermediate interval where both phases coexisted without transformation [9]. This allows to investigate if the theoretical model, calculated from data when the sample is in a monophasic state, is suitable to describe data in the coexistence interval. From specific heat, latent heat and dielectric-constant measurements, we show in this letter that a 2-4-6 Landau potential describes whole sets of data, including those corresponding to the coexistence interval.

Experimental. - The sample was prepared at the Institute of Physics, Adam Mickiewicz University, Poznan (Poland). The degree of deuteration was estimated to be $90 \%$ from the relation given by Gesi [4]. The thickness of the sample was $2.55 \mathrm{~mm}$ and the mass $0.35 \mathrm{~g}$. The main faces, with a pseudo-hexagonal cross-section of $71 \mathrm{~mm}^{2}$, were prepared perpendicular to the $b$-axis and gold electrodes were evaporated on them. Optical observations showed an internal crack perpendicular to the main faces across the sample.

The calorimetric measurements were performed by means of a high-resolution conduction calorimeter, which has been described in detail [13]. The absolute value of the specific heat is obtained by integrating the electromotive force given by two heat fluxmeters when the temperature of the sample changes as a consequence of the superposition of a long-periodic serial of square thermal pulses to a heating or cooling ramp [14]. We obtained two sets of data which correspond to the dissipation and non-dissipation semiperiods of every square pulse, $c_{d}$ and $c_{r}$, respectively. Both sets of data coincide except in the temperature interval where a latent heat is involved. In this case, $c_{d}$ and $c_{r}$ become different and show a very anomalous behaviour due to the latent heat effect in the measurement process. This fact permits the determination of the temperature interval where the latent heat is present [10].

In a second experiment, we use the equipment as a very sensitive DTA device. The high number of thermocouples forming the fluxmeter allows to use a temperature variation rate similar to that used in heat capacity measurements. The latent heat is evaluated by the comparison of the DTA trace with specific heat data following the method described in ref. [15].

The dielectric constant was measured using a capacitance bridge ESI-SP 5400 at a frequency of $1 \mathrm{kHz}$ in a cell different to that used in the thermal-properties measurements.

Results. - The specific heat was measured on cooling and heating at a scanning temperature rate of $0.03 \mathrm{~K} \mathrm{~h}^{-1}$. The quasiestatic variation of temperature during the experiment allows to determine precisely the anomaly of the specific heat (a point of specific heat is obtained every $0.006 \mathrm{~K}$ and the temperature increment of the sample during the measurement process is evaluated to be $0.01 \mathrm{~K}$ ). In fig. 1 we represent the specific heat excess $\Delta c$ obtained on cooling (a) and on heating (b) for dissipation and non-dissipation branches in a very narrow temperature interval around the transition temperature (data in a larger temperature interval have already been published [9]). The baseline to determine the specific heat excess has been taken as a linear extrapolation of the high-temperature data.

The DTA traces $\phi_{D}$ on cooling and on heating have been represented in fig. 2 together with the DTA traces calculated from the specific heat data $\phi_{c}[15]$.

The curves in figs. 1 and 2 show a similar behaviour: we distinguish five regions labelled from $\mathrm{A}$ to $\mathrm{E}$. In regions $\mathrm{A}, \mathrm{C}$ and $\mathrm{E}, \Delta c_{r}$ and $\Delta c_{d}$ show a regular behaviour and $\phi_{D}$ and 

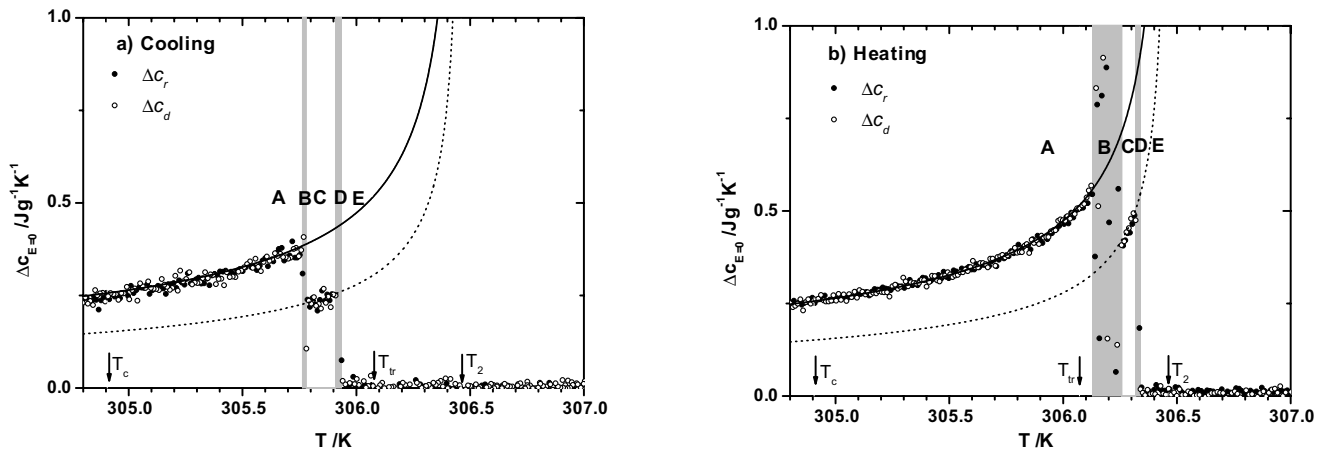

Fig. 1 - Specific heat excess for dissipation branch (open circles) and for non-dissipation branch (full circles). The continuous line represents the Landau prediction when the sample is completely in the ferroelectric phase. The dotted line is the prediction when the $59 \%$ of the sample is in the ferroelectric phase. The characteristic temperatures $T_{c}, T_{t r}$ and $T_{2}$ are also included.

$\phi_{c}$ coincides, indicating that there is not latent heat effect. In regions B and D (shaded in the figures), $\phi_{D}$ is higher than $\phi_{c}$ and $\Delta c_{r}$ and $\Delta c_{d}$ shows an anomalous behaviour including some data which are out of scale in fig. 1 . In these regions, the phase transition takes place and the effect of the latent heat is present.

The existence of the intermediate region $\mathrm{C}$ is attributed to the presence of the crack in the sample. One of the pieces of the sample undergoes the phase transition (in region $\mathrm{D}$ on cooling and in region B on heating) while the other remains untransformed. After that, both parts of the sample modify their temperature without transformation so that the specific heat data correspond to a system with two phases which evolve independently. Finally, the portion of the sample which remained untransformed undergoes the change of phase (in region B on cooling and in region D on heating) and the transition is completed.

The enthalpy variation of each region is calculated by integration of the DTA trace with respect to the baselines shown in fig. 2. The latent heat is evaluated to be $L_{c}=1.32 \pm 0.02 \mathrm{~J} \mathrm{~g}^{-1}$ on cooling run and $L_{h}=1.08 \pm 0.02 \mathrm{~J} \mathrm{~g}^{-1}$ on heating run. This difference is due to the fact that the specific heat on heating reaches higher values than on cooling as a consequence of the
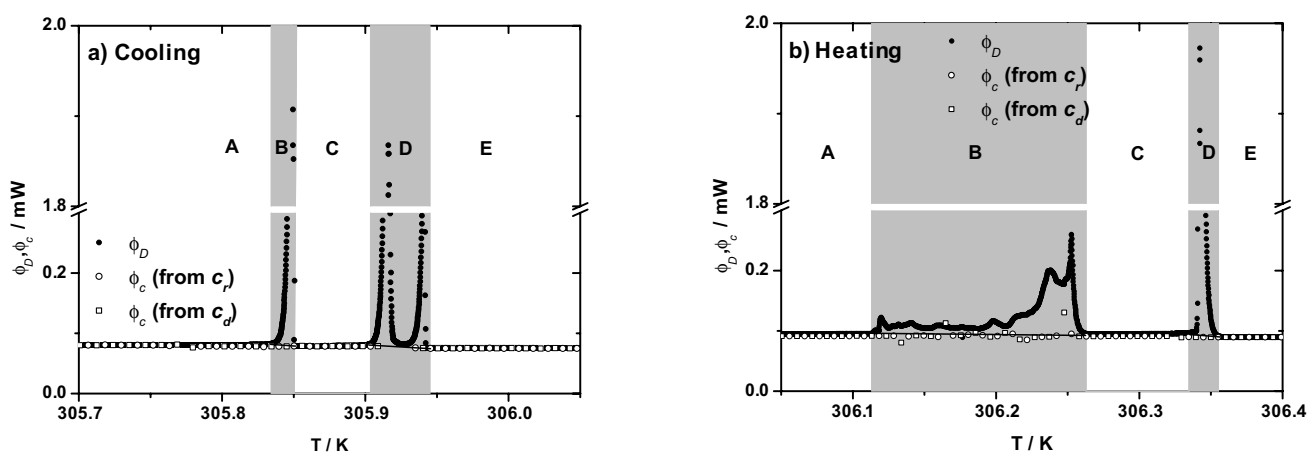

Fig. 2 - DTA traces $\phi_{D}$ on cooling and on heating (full circles) and heat flux calculated from specific heat data $\phi_{c}$ (open circles and open squares). The baseline to determine the latent heat is included. 

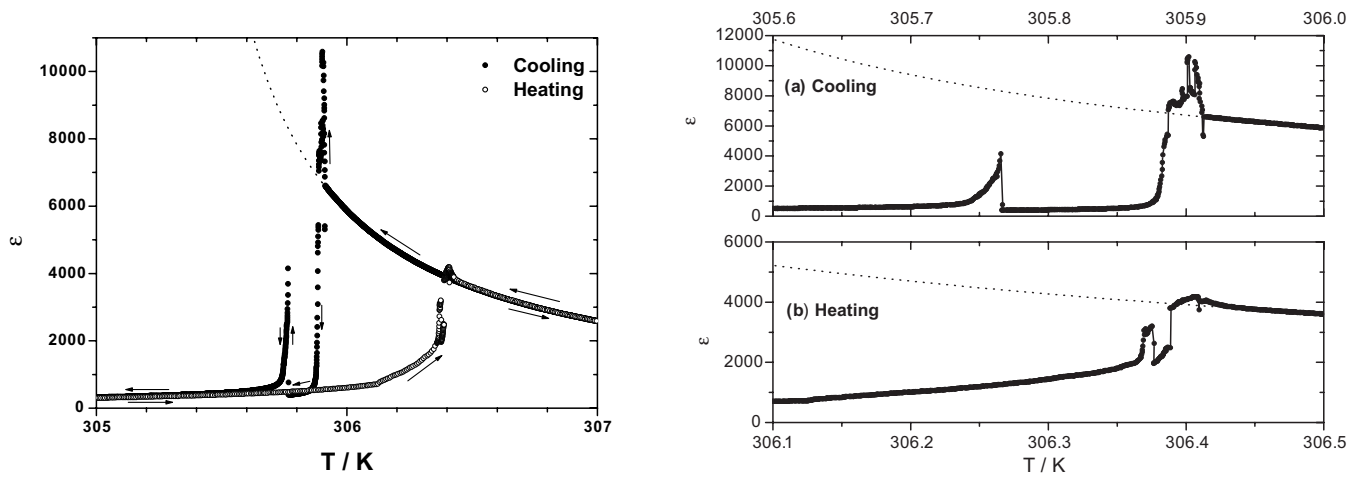

Fig. 3 - Dielectric constant on cooling (full circles) and on heating (open circles) and fitted Curie law (discontinuous line). The graphs on the right show $\varepsilon$ in a narrower temperature interval.

thermal hysteresis as we will discuss below. Nevertheless, we have shown that the enthalpy balance between the ferroelectric and the paraelectric phase is fulfilled [9].

From the evaluation of the proportion between the enthalpy variation in regions $\mathrm{B}$ and $\mathrm{D}$, we found that the transition is blocked in region $\mathrm{C}$ with a proportion of $59 \%$ of the sample in the ferroelectric phase and the remaining $41 \%$ in the paraelectric phase.

The dielectric constant $\varepsilon$ has been represented in fig. 3, where the temperature interval around the transition temperature has also been enlarged to observe in detail the kinetics of the transformation. In paraelectric phase, the Curie law, $\varepsilon=\frac{C_{C W}}{T-T_{c}}$ is fulfilled with $C_{C W}=4700 \mathrm{~K}$ (discontinuous lines in fig. 3). The thermal hysteresis is higher than that found in the thermal measurements, which is attributed to the fact that the dielectric measurements have been carried out in a different device and at a higher-temperature variation rate $\left(0.5 \mathrm{~K} \mathrm{~h}^{-1}\right)$. Despite this difference, there are similar features to specific heat and DTA traces since the transition also takes place in two stages which are clearly seen on cooling in fig. 3(a).

It has been shown that the behaviour of the dielectric constant in a first-order phase transition provides information about the kinetics of the phase transition [16-19]. Let us consider the paraelectric-ferroelectric phase transition. The temperature dependence of $\varepsilon$ deviates from the Curie law when the nuclei of the ferroelectric phase are present in the paraelectric phase. It is clearly observed in fig. 3(a) since $\varepsilon$ increases rapidly. The maximum value that $\varepsilon$ reaches depends on the dynamics of the domain structure and changes for successive paraelectric to ferroelectric phase transitions. The dielectric constant relaxes in the temperature interval where ferro and paraphase coexist without phase transformation (region C in DTA trace). A second sharp anomaly in $\varepsilon$ is found when the temperature continues decreasing, which corresponds to the transformation of the second part of the sample. Similar features are observed in ferroelectric-paraelectric phase transformation (fig. 3(b)) but, in this case, are not so significant since the values of $\varepsilon$ on cooling are higher than on heating as a consequence of the instability of the ferroelectric domains.

Landau potential. - The 2-4-6 Landau expansion in terms of the order parameter $P$, $\Delta G=\frac{1}{2} \alpha\left(T-T_{c}\right) P^{2}+\frac{1}{4} \beta P^{4}+\frac{1}{6} \gamma P^{6}$, predicts that $\Delta c$ in the ferroelectric phase is given by

$$
\left(\frac{T}{\Delta c}\right)^{2}=\frac{4 \beta^{2}}{\alpha^{4}}+\frac{16 \gamma}{\alpha^{3}}\left(T_{c}-T\right) .
$$


In a first-order phase transition, it is more convenient to introduce three different temperatures which characterise a first-order phase transition: $T_{c}, T_{t r}$ and $T_{2}$ [20,21]. The lowest of them, $T_{c}$, is the lowest temperature for which $P=0$ is a minimum in $G$. $T_{t r}$ is the temperature at which $G$ for the ferrophase and the paraphase are equal and so is the temperature at which an equilibrium transformation should occur. $T_{2}$ is the highest temperature for which the ferroelectric phase may exist. These temperatures are related by

$$
T_{2}-T_{c}=4\left(T_{2}-T_{t r}\right)=\frac{\beta^{2}}{4 \alpha \gamma} .
$$

$T_{2}$ and $T_{c}$ limit theoretically the region where the paraphase and the ferrophase may coexist. The extent of this interval $d=T_{2}-T_{c}$ represents the maximum thermal hysteresis although the experimental value is generally lower than the theoretical one.

The latent heat in Landau theory is theoretically defined from the step which the entropy excess $\Delta S$ would show if the transition took place at $T_{t r}$ (in equilibrium of phases)

$$
L=T_{t r} \Delta S\left(T=T_{t r}\right)=\frac{3 \alpha \beta T_{t r}}{8 \gamma} .
$$

It is interesting to note that, in spite of the use of a low-temperature variation rate to perform the experiments, thermal hysteresis appears. The transition does not actually take place in equilibrium of phases at $T_{t r}$. On cooling the paraelectric phase is a metastable state between $T_{t r}$ and $T_{c}$ and the transition can be produced at any temperature within this interval. In a similar way, on heating, the ferroelectric phase is a metastable state between $T_{t r}$ and $T_{2}$ and the transformation can occur at any temperature within this interval. As a consequence, the transition on heating will take place at temperatures higher than on cooling and the characteristic first-order step in the order parameter at the transition will be lower than on cooling and also the latent heat. When the latent heat is measured, we obtain two different values, one on heating $L_{h}$ and one on cooling $L_{c}$. The theoretical latent heat $L$ will lie between both and we will take it as the mean value of the measured values on heating and on cooling, giving $L=1.20 \mathrm{Jg}^{-1}=480 \mathrm{~J} \mathrm{~mol}^{-1}$. The uncertainty that this election would imply in the determination of the coefficient $\beta$ will always be lower than that obtained if we had taken $L$ as the cooling value $L_{c}$ or as the heating value $L_{h}$, as is generally made. The value of $T_{t r}$ will be calculated below.

The coefficient of the Landau potential $\alpha$ is obtained from the dielectric data. Using the value of $C_{C W}$, we obtain $\alpha=2.40 \times 10^{7} \mathrm{~J}^{-1} \mathrm{~V}^{2} \mathrm{mK}^{-1}$ and $T_{c}=305.20 \mathrm{~K}$. We will not use this value for $T_{c}$ because the measurements of $\varepsilon$ have been carried out in a cell different to that used for the measurement of the thermal properties and at a different temperature variation rate.

Equation (1) can be rewritten in terms of the characteristic temperature $T_{2}$ :

$$
\left(\frac{T}{\Delta c}\right)^{2}=\frac{16 \gamma}{\alpha^{3}}\left(T_{2}-T\right) .
$$

In fig. 4 we represent $\left(\frac{T}{\Delta c}\right)^{2} v s$. temperature for data on region A. The data falls in a straight line which intercepts the temperature axis at $T_{2}=306.46 \mathrm{~K}$ and whose slope is evaluated to be $9.10 \times 10^{5} \mathrm{~K}^{3} \mathrm{~J}^{-2} \mathrm{~g}^{-2}$. Taking into account that this slope is given by $\left(16 \gamma / \alpha^{3}\right)$ and using the value of the density $\left(\rho=1.845 \mathrm{~g} \mathrm{~cm}^{-3}\right)$ and $\alpha$ previously determined, the coefficient $\gamma$ is calculated: $\gamma=2.32 \times 10^{14} \mathrm{~J}^{-5} \mathrm{~V}^{6} \mathrm{~m}^{9}$.

From eqs. (2) and (3) and introducing the calculated value for $L=1.20 \mathrm{Jg}^{-1}=2.21 \times$ $10^{6} \mathrm{~J} \mathrm{~m}^{-3}$, we obtain $\beta=-1.8 \times 10^{11} \mathrm{~J}^{-3} \mathrm{~V}^{4} \mathrm{~m}^{5}, T_{t r}=306.07 \mathrm{~K}$ and $T_{c}=304.91 \mathrm{~K}$. The 


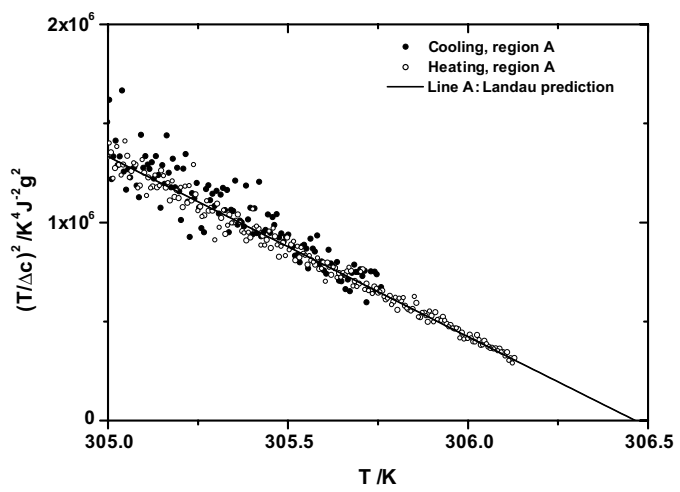

Fig. $4-\left(\frac{T}{\Delta C}\right)^{2}$ vs. temperature in region A on cooling (full circles) and heating (open circles).

maximum thermal hysteresis is given by $d=T_{2}-T_{c}=1.55 \mathrm{~K}$. The thermal hysteresis found in thermal and dielectric measurements are $0.2 \mathrm{~K}$ and $0.55 \mathrm{~K}$ respectively, which are lower than $d$.

We have included in fig. 1 the prediction for the specific heat excess from the Landau potential, indicating $T_{c}, T_{t r}$ and $T_{2}$. Data corresponding to region A where the sample is completely in the ferroelectric phase are fully explained by the Landau potential. It is also interesting to note that both stages of the transition on cooling takes place between $T_{t r}$ and $T_{c}$ and on heating between $T_{c}$ and $T_{2}$ in full agreement with the theoretical model.

It has been stated above that in the temperature interval which corresponds to region $\mathrm{C}$, ferroelectric and paraelectric phases coexist in the sample without transformation and without effect from the latent heat. In this region, the molar fraction of the ferroelectric phase is $x_{\text {ferro }}=0.59$. According to this, the specific heat excess of the sample in this coexistence interval should be given by $\Delta c=x_{\text {ferro }} \Delta c_{\text {ferro }}$ because $\Delta c$ in the paraelectric phase is nil. In fig. 1 , we also represent $x_{\text {ferro }} \Delta c_{\text {ferro }}$, where $\Delta c_{\text {ferro }}$ is obtained from the 2-4-6 Landau potential determined from the analysis of regions $\mathrm{A}$ and $\mathrm{E}$, which correspond to states where the sample is completely in the ferroelectric and in the paraelectric phase, respectively. A very good agreement between data in region $\mathrm{C}$ and Landau predictions is found.

In conclusion, the phase transition in a $90 \%$ deuterated TGSe has been investigated by thermal and dielectric measurements. Irrespective of the heating or cooling regime, due to an internal crack the phase transition occurs in two stages separated by an interval where both phases coexist but without latent heat contribution. We have analised if there could be a temperature gradient in the sample which could make the temperature of one of the parts be always higher. Each main face of the sample is in thermal contact with the end of a fluxmeter, which is an isothermal plane. The temperature difference between both faces of the sample has been evaluated to be lower than $0.01 \mathrm{~K}$. However, it cannot justify that one of the parts of the sample is always at higher temperatures since the internal crack is perpendicular to the main faces of the sample and, hence, to isothermal planes of the assembly.

It is a fact that the nucleation of the ferroelectric phase starts in the $59 \%$ part of the sample when cooling and the transformation to the paraelectric phase begins in the $41 \%$ part when heating. In both cases, the crack acts as a lateral barrier for the phase front. A possible explanation could be related to the fact that the transition temperature decreases when a uniaxial pressure is applied along the ferroelectric axis [22] and the fact that the uniaxial pressure on the main faces of the sample could be non-uniform. It could be due to a lack of parallelism between the faces of the sample and the ends of the fluxmeters, which press the 
sample to support it. We must point out that the second stage of the transformation is very sharp on heating (region D) and on cooling (region B), because the heat flux is equivalent to an extremely short heat pulse. It is an evidence of the metastable state produced by the crack. If the crack were not present the transition took place in the entire sample, giving one peak, and if two separated pieces of the sample were under similar conditions, the two stages of the transformation would show a similar behaviour.

$$
* * *
$$

We thank Prof. Koralewski for supplying the sample. This work has been supported by the project FIS2005-02444 of Spanish DGICYT.

\section{REFERENCES}

[1] Jona F. and Shirane G., Ferroelectric Crystals (New York, Pergamon) 1962.

[2] Matthias B. T., Miller C. E. and Remeika J., Phys. Rev., 104 (1956) 849.

[3] Romero F. J., Gallardo M. C., Jiménez J., Koralewski M., Czarnecka A. and del Cerro J., J. Phys. Condens. Matter, 16 (2004) 7637.

[4] Gesi K., J. Phys. Soc. Jpn., 41 (1976) 565.

[5] Aragó C. and Gonzalo J. A., J. Phys. Condens. Matter, 12 (2000) 3737.

[6] Aragó C. and Gonzalo J. A., Ferroelectrics Lett., 27 (2000) 83.

[7] Song Y. W., Taraskin S. A. and Strukov B. A., J. Korean Phys. Soc., 27 (1994) S74.

[8] Song Y. W., Kim J. C., You I. K. and Strukov B. A., Mat. Res. Bull., 35 (2000) 1087.

[9] Romero F. J., Gallardo M. C., Czarnecka A., Koralewski M. and del Cerro J., Thermal and kinetic study of the ferroelectric phase transition in deuterated triglycine selenate, to be published in J. Therm. Anal. Calorim. (2006) DOI:10.1007/s10973-005-7444-7.

[10] del Cerro J., Martín-Olalla J. M. and Romero F. J., Thermochim. Acta, 149 (2003) 401.

[11] Romero F. J., Gallardo M. C., Jiménez J., del Cerro J. and Salje E. K. H., J. Phys. Condens. Matter, 12 (2000) 4567.

[12] Delgado-Sánchez J. M., Martín-Olalla J. M., Gallardo M. C., Ramos S., Koralewski M. and del Cerro J., J. Phys. Condens. Matter, 17 (2005) 2645.

[13] Gallardo M. C., Jiménez J. and del Cerro J., Rev. Sci. Instrum., 66 (1995) 5288.

[14] del Cerro J., J. Therm. Anal., 34 (1988) 335.

[15] del Cerro J., Romero F. J., Gallardo M. C., Hayward S. A. and Jimenez J., Thermochim. Acta, 343 (2000) 89.

[16] Bornarel J. and Cach R., Ferroelectrics, 124 (1991) 345.

[17] Bornarel J. and Cach R., Phys. Rev. B, 60 (1999) 3806.

[18] Kvitek Z. and Bornarel J., J. Phys. Condens. Matter, 12 (2000) 7819.

[19] Bornarel J. and Cach R., J. Phys. Condens. Matter, 15 (2003) 4371.

[20] Salje E. K. H., Phase Transitions in Ferroelastic and Coelastic Materials (Cambridge University Press) 1990.

[21] Hayward S. A., Romero F. J., Gallardo M. C., del Cerro J., Gibaud A. and SalJe E. K. H., J. Phys. Condens. Matter, 12 (2000) 1133.

[22] Koralewski M., Stankowska J., Iglesias T. and Gonzalo J. A., J. Phys. Condens. Matter, 8 (1996) 4079. 